\title{
A Modified Code Tracking Loop \\ for Direct-Sequence Spread-Spectrum Systems \\ on Frequency-Selective Fading Channels
}

\author{
Jia-Chin Lin and Lin-Shan Lee \\ Department of Electrical Engineering \\ National Taiwan University \\ Taipei, Taiwan, Republic of China
}

\begin{abstract}
A modified fully-digital code tracking loop is proposed in this paper for direct-sequence spread-spectrum signaling on a frequency-selective fading channel. A data-modulated channel estimator is used to cope with the time-varying Rayleigh fading effect and the data modulation effect, and extract the desired error signal from each path independently in the multipath environments. By taking advantage of the inherent diversity with the maximal ratio combining (MRC) or a proposed Even/odd maximal ratio combining (EMRC) technique, this modified code tracking loop can avoid the problem due to the drift or flutter effects of the error characteristics, and provide better performance on frequency selective fading channels. Extensive computer simulation has verified the analysis and indicated very attractive behavior of the proposed digital tracking loop.
\end{abstract}

\section{IntroduCtion}

G UBSTANTIAL efforts have been made in the $S$ code tracking problem for spread spectrum communications [1], [2], though most of the analyses were in the context of analog implementation and AWGN channels. However, very often the frequency-selective fading [3] in addition to AWGN could seriously destroy the tracking capabilities of the conventional delay-locked loops. The very successful joint estimators for multipath effects, and code delay based on extended Kalman filter (EKF) [4], [5], [6] can very well take the multipath effects into consideration in advance. However, since CDMA wireless communication systems usually operate in seriously noisy environments, it is practically not easy for the estimators to function with heavy computation load. In this paper, a fully-digital, noncoherent, modified code tracking loop is proposed, which can operate on bandlimited DS/SS systems over frequency-selective fading channels.

\section{System Description of the Proposed Modified Code Tracking LoOP}

The modulator and demodulator schemes of a bandlimited DS/SS M-PSK system have been well known [7]. To describe in detail the operations in the modified digital delay-locked loop (DDLL) proposed in this paper, a complete block diagram of the loop is sketched in Figure 1. The tapped delay line model [3], [8] is applied as frequency-selective fading channel in this paper. The complex representation of the baseband signal at the output of the chip matched filter is

$$
\tilde{r}(t)=e^{j \theta} \sum_{i=0}^{\infty} d_{i} \sum_{n=0}^{L-1} a_{n}(t) P^{\prime}\left(t-n T_{\mathbf{c}}-i T_{b}\right)+\tilde{n}(t)
$$

where $P^{\prime}(t)=\sum_{k=0}^{M-1} c_{k} g\left(t-k T_{c}\right)$ is the chip-shaped PN sequence, $L$ is the number of resolvable paths, $\theta$ denotes the carrier phase error introduced by the front-end noncoherent down-conversion process, $d_{i}$ is the $\mathrm{i}$-th information-bearing M-PSK symbol, $c_{k}$ is the $\mathrm{k}$-th chip value of the PN sequence, $T_{b}$ and $T_{c}$ are the symbol interval and chip duration respectively, $M=\frac{T_{b}}{T_{c}}$ is the processing gain, $g(t)$ is the overall chip shape. Without loss of generality, it is assumed for simplicity here the period of the PN code is exactly $M$. The signal $\tilde{r}(t)$ is sampled at the instants $t_{k}=\left(k+\varepsilon_{k}\right) T_{c}$ and $t_{k+\frac{1}{2}}=\left(k+\varepsilon_{k}+\frac{1}{2}\right) T_{c}$, where $\varepsilon_{k}$ is the kth normalized chip timing error, to produce the two parallel sequences: integer-instant samples $\tilde{r}_{k}=\tilde{r}\left(t_{k}\right)$ and half-integer-instant samples $\tilde{r}_{k+\frac{1}{2}}=\tilde{r}\left(t_{k+\frac{1}{2}}\right)$.

\section{A. Channel Estimation}

The integer-instant samples $\tilde{r}_{k}$ are fed to the data-modulated channel estimator. Assume first 
the code acquisition process has been achieved. The channel estimation process is performed by means of channel sounding using the datamodulated PN sequence, faded by the channel, cross-correlated with the local replica of the PN sequence in the receiver, and feeding to the Accumulate/Reset (A/R) filters (similar to the Integrate/Dump in analog implementation). The A/R filters collect the outputs of the cross-correlators, and dump the lowpass components as the estimates of the channel tap weights modulated by information-bearing symbols at the end of each symbol duration. The estimate of the $n$-th channel path coefficient (i.e., the output of the n'-th $\mathrm{RAKE}$-branch estimate) is given by

$$
\begin{aligned}
& \hat{C}_{[k]_{M}}^{n^{\prime}}(\varepsilon)=\frac{1}{M} \sum_{l=M\left([k]_{M}-1\right)+n^{\prime}}^{M \cdot[k]_{M}-1+n^{\prime}} \quad\left(\tilde{r}_{l} \cdot c_{l-n^{\prime}}\right) \\
& 0 \leq n^{\prime} \leq L-1
\end{aligned}
$$

where $[k]_{M}$ denotes the integer part of $k / M$. These estimates of the channel tap weights depend on the timing error, because the channel souriding process inherently employs the cross-correlation between the received signal and the only code-acquired (with timing error) local PN sequence.

\section{B. RAKE-Based Early-Late Structure}

The half-integer-instant samples $\tilde{r}_{k+1 / 2}$ are buffered by a queue with length equal to the processing gain, $M$, because the channel estimator should update those data-modulated channel coefficient estimates just at the end of each symbol duration. We need to introduce a delay of one symbol interval (i.e., $\mathrm{M}$ chips) before the half-integerinstant samples enter the early-late structure, so that those complex channel coefficients by which the samples entering the early-late structure are to be corrupted can be properly estimated by the channel estimator. The early-late structure proposed here can be also seen in Figure 1, which is based on the RAKE structure to exploit the multipath diversity. The integration is therefore inherently performed by the number-controlled oscillator (NCO) with the following loop equation

$$
\varepsilon_{k+1}=\varepsilon_{k}-\gamma e_{k}
$$

where $\gamma$ is the NCO sensitivity; and $e_{k}$ is the compound error signal obtained at MRC or EM$\mathrm{RC}$ based on the data-modulated channel-faded branch error signals, $z_{k}^{n^{\prime}}$, produced from the earlylate structure. The average loop error characteristics are defined as

$$
S(\varepsilon) \equiv<E\left\{e_{k} \mid \varepsilon_{k}=\varepsilon, \quad \forall k\right\}>
$$

where the operator $\langle\cdot\rangle$ denotes time-averaging.

In each branch of the early-late structure, the incoming delayed half-integer-instant samples $\tilde{r}_{k-M+1 / 2}$ are cross-correlated with the delayed replica of the code difference streams, $c_{\Delta}^{n^{\prime}}(k)=$ $\left[c_{k-n^{\prime}}-c_{k+1-n^{\prime}}\right]$, for $0 \leq n^{\prime} \leq L-1$. After going through the branch filters, $h_{k}$, the data-modulated channel-faded branch error signal, $z_{k}^{n^{\prime}}$, are generated,

$$
z_{k}^{n^{\prime}}=\tilde{r}_{k-M+\frac{1}{2}} \times c_{\Delta}^{n^{\prime}}(k) \otimes h_{k} \quad 0 \leq n^{\prime} \leq L-1
$$

$z_{k}^{n^{\prime}}$ here is similar to the error signal of the conventional DLL on the single-path AWGN environments, besides here they are corrupted by the channel tap weights, the information-bearing symbol and the non-negligible interference introduced from the residual cross-correlation between the adjacentpath signals and the code applied in this branch.

\section{C. $M R C$ and EMRC}

\section{C.1 MRC}

The data-modulation effect and the channel multipath fading effect on the error signal at the n'th RAKE branch, $z_{k}^{n^{\prime}}$, need to be compensated for. The compound error signal, $e_{k}$, can be obtained from $z_{k}^{n^{\prime}}$ and the data-modulated channel estimates, $\hat{C}_{[k]_{M}}^{n^{\prime}}(\varepsilon)$, using maximal ratio combining (MRC) criterion,

$$
e_{k}=\sum_{n^{\prime}=0}^{L-1} \operatorname{Re}\left\{e_{k}^{n^{\prime}}\right\}=\sum_{n^{\prime}=0}^{L-1} \operatorname{Re}\left\{z_{k}^{n^{\prime}} \cdot \hat{C}_{[k]_{M}}^{n^{\prime *}}(\varepsilon)\right\}
$$

The carrier phase error $e^{j \theta}$ introduced by the frontend noncoherent down-conversion process and the data modulation effect on $z_{k}^{n^{\prime}}$ are thus effectively compensated for by the complex conjugate of the data-modulated channel estimate on each RAKE branch. The branch error signals suffer from the 
self-interference generated by the adjacent branches of the RAKE structure. For a certain propagation path, some typical branch error signal detected on the corresponding branch looks like the S-curve of a conventional DLL in a single-path AWGN environment. However, the residual crosscorrelation, which is beyond the half chip duration, is inevitably detected and causes some undesired error signals on the preceding and succeeding RAKE branches, respectively. The undesired self-interference naturally blocks the desired error signals. As a result, the compound error characteristics for the proposed modified code tracking loop with MRC are the superposition of the effective error characteristics offered by all the channel propagation paths. The self-cancellation effect caused by the self-interference coming from adjacent branches is the primary drawback of MRC. A modified combination scheme, referred to here as the Even/odd maximal ratio combining (EM$\mathrm{RC}$ ) criterion, is therefore proposed in this paper to solve this problem, and it can also reduce the hardware complexity.

\section{2 EMRC}

The RAKE branches are first divided into two sets, the odd and even branches. The branch error signals for each set are individually combined by means of MRC to produce odd-branch and evenbranch compound error signals, $e_{k}^{o}$ and $e_{k}^{e}$, respectively,

$$
\begin{aligned}
& e_{k}^{o}=\sum_{n^{\prime}: \text { odd }} \operatorname{Re}\left\{z_{k}^{n^{\prime}} \cdot \hat{C}_{[k]_{M}}^{n^{\prime *}}(\varepsilon)\right\} \\
& e_{k}^{e}=\sum_{n^{\prime}: \text { even }} \operatorname{Re}\left\{z_{k}^{n^{\prime}} \cdot \hat{C}_{[k]_{M}}^{n^{\prime *}}(\varepsilon)\right\}
\end{aligned}
$$

Between these two compound error signals, the one with higher absolute value is selected as the input to the loop filter,

$$
e_{k}=\arg \max _{\left\{e_{k}^{e}, e_{k}^{e}\right\}}\left\{\left|e_{k}^{o}\right|,\left|e_{k}^{e}\right|\right\}
$$

This scheme can in fact be implemented with only half of the hardware complexity of MRC, because the compound error signal for the even branches can actually be obtained from the same hardware for the odd branches. The only difference is the one chip duration (i.e., one sample) delay introduced into both inputs of the channel estimator and the early-late structure. Therefore, the hardware for the odd branches simply processes sequentially to produce $e_{k}^{o}$ and $e_{k}^{e}$ alternatively, and then the larger one out of any two consecutive outputs is selected to be the input of the loop filter.

Although the proposed EMRC discussed above doesn't really confine the range of S-curve in each branch and the self-interference still appear in adjacent branches, in principle the time delay between any two consecutive RAKE branches in either of the two individual sets, even or odd, is twice of that between any two consecutive channel paths. Because only one output out of these two sets is selected to be fed to the loop filter, this scheme effectively avoids the self-cancellation problem.

\section{Simulation Results}

The modified code tracking loop presented above has been simulated on a computer to evaluate the error characteristics, noise performance and loop robustness based on Monte Carlo methods. The simulation parameters are given below.

Modulation: 8-PSK

PN code: m-sequence with generating polynomial $g(x)=1+x^{3}+x^{7}$

Chip Shaping: square root raised cosine with roll-off factor $\alpha=0.35$

Chip Rate: $\quad \frac{1}{T_{c}}=1.27 M$ chips $/ \mathrm{sec}$

Symbol Rate: $\quad \frac{1}{T_{b}}=10 K$ symbols $/ \mathrm{sec}$

Sampling Rate: 16 samples per chip period Channel \#1: four paths with equal power, each path is modeled with an independent Jakes' fading model with maximal Doppler frequencies 8,80,10,100 $\mathrm{Hz}$

RAKE: four branches with branch filter bandwidth $B_{b}=\frac{1}{T_{b}}$

Loop Filter: normalized bandwidth:

$$
B_{L} T_{c}=2.5 \times 10^{-2}
$$

From the above, multipath introduces significant timing errors, and the track jitter and MTLL simulation for the conventional DLL on the theoretical multipath channels is not needed, because the conventional DLL is well known to be vulnerable to multipath channel effects.

Figure 2-(1) presents the S-curves of the modified code tracking loops with MRC and EMRC on 
channel \#1. It is obvious from Figure 2-(1) that for the S-curves of these modified code tracking loops, with stable locked point at $\varepsilon=0$, there exist no phenomena of drift or flutter of the locked point as observed previously for conventional DLLs [3]. The S-curve for MRC is apparently lower than that for single-path environments because MRC introduces inevitable self-cancellation. Similarly, higher error characteristics can be detected for the loop with EMRC than that with MR$\mathrm{C}$, though lower than that for single-path environments; because the EMRC scheme can minimize the adjacent-branch self-cancellation. From the simulation, the mean squared timing errors for M$\mathrm{RC}$ and EMRC schemes under different SNR conditions are also obtained and shown in Figure 2-(2). We can see from this figure that the mean squared timing errors for the EMRC scheme is less than that for MRC. This indicates that EMRC provides more stable steady-state performance.

The mean time to lose lock (MTLL) is very important for code tracking loop, specially in the low SNR conditions. The MTLL for the proposed modified code tracking loop is defined as the mean time that all branches of the RAKE structure remain synchronized. Figure 2-(3) presents the simulated results for the MTLL of the proposed modified code tracking loops with either MRC or EMRC scheme, under different SNR conditions. We can also see that the modified code tracking loop with EMRC always have longer MTLL than that with MRC. This makes sense, because the larger area under the S-curve of EMRC for positive $\varepsilon$ implies higher escape energy, which can support longer MTLL [9], $[10]$.

\section{CONCLUSION}

A modified fully-digital code tracking loop is proposed in this paper for direct sequence spreadspectrum signaling on frequency-selective fading channels. Because the data-modulated channel estimator can cope with the time-varying Rayleigh fading effect and the data modulation effect on the error signals, in addition to exploiting the multipath diversity by means of RAKE structure, the modified code tracking loop with either MR$\mathrm{C}$ or EMRC is shown to provide very good performance on frequency-selective fading channels in the sense of jitter performance and MTLL measurement. Moreover, an EMRC scheme is proposed to solve the adjacent-branch self-cancellation in the MRC scheme. The simulation results of the mean squared timing error and MTLL show that the EMRC scheme certainly has more stable noise performance, better loop robustness, and lower hardware complexity.

\section{ACKNOWLEDGEMENT}

This research was supported by the National Science Council, Taiwan, ROC under the contract NSC 86-2213-E002-025.

\section{REFERENCES}

[1] M. Simon, T. Omura, R. Scholtz, and K. Levitt, Spread Spectrum Communication, vol. I, vol. II, and vol. III, Rackvill, MD: Compute Science Press, 1985.

[2] R. A. Yost and R. W. Boyd, "A modified PN code tracking loop: Its performance analysis and comparative evaluation," IEEE Trans. Commun., Vol. 30, pp. 1027-1036, May 1982.

[3] W.-H. Sheen and Gordon L. Stuber, "Effects of Multipath Fading on Delay-Locked Loops for Spread Spectrum Systems," IEEE Trans. Commun., Vol. 42, pp. 1947-1956 Feb./March/April 1994.

[4] R. A. Iltis, "Joint Estimation of PN Code Delay and Multipath Using the Extended Kalman Filter," IEEE Trans. Commun., Vol. 38, pp. 1677-1685, October 1990.

[5] R. A. Itis and A. W. Fuxjaeger, "A Digital DS SpreadSpectrum Receiver with Joint Channel and Doppler Shift Estimation," IEEE Trans. Commun., Vol. 39, pp. 12551267, August 1991.

[6] R. A. Iltis, "An EKF-Based Joint Estimator for Interference Multipath, and Code Delay in a DS Spread-Spectrum Receiver," IEEE Trans. Commun., Vol. 42, pp. 1288-1299, Feb./March/April 1994.

[7] R. D. Gaudenzi, M. Luise and R. Viola, "A Digital Chip Timing Recovery Loop for Band-Limited Direct-Sequence Spread-Spectrum Signals," IEEE Trans. Commun., Vol. 41, pp. 1760-1769, Nov. 1993.

[8] John G. Proakis, Digital Communications, NY: MacGraw Hill, 1989.

[9] A. Wilde and U. P. Bernhard, "Mean Time to Lose Lock for a Second-Order Extended Tracking Range Delay-Locked Loop," Proc. of PIMRC'95, pp. 1000-1004, 1995.

[10] A. L. Welti and B. Z. Bobrovsky, "Mean Time to Lose Lock for the "Langevin"-Type Delay-Locked Loop," IEEE Trans. Commun., Vol. 42, pp. 2526-2530, August 1994.

[11] W.-H. Sheen and Gordon L. Stuber, "A New Tracking Loop for Direct Sequence Spread Spectrum Systems on Frequency-Selective Fading Channel," Proc. of ICC'95, pp. 1364-1368 1995. 

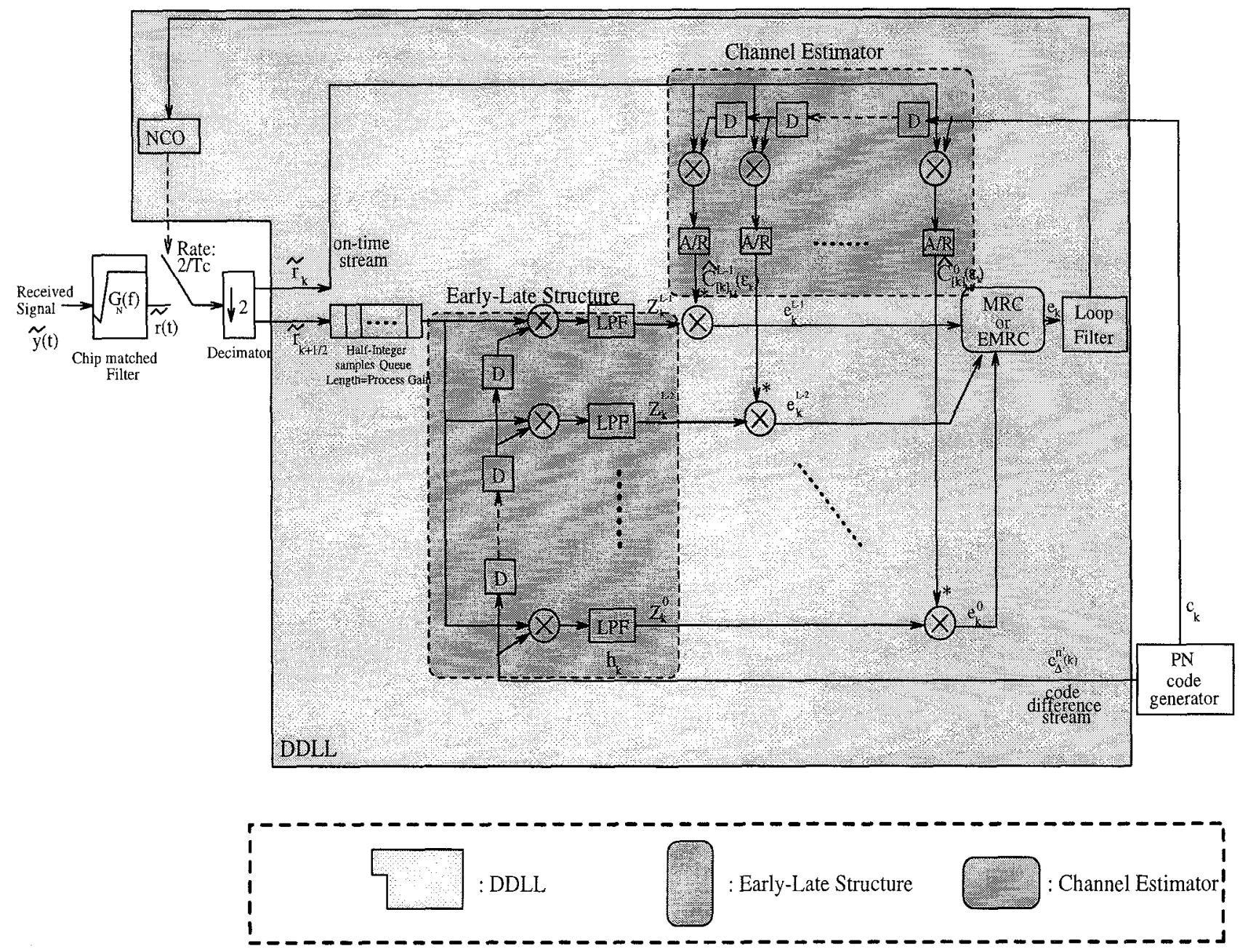

Fig. 1. Block diagram of the modified DDLL

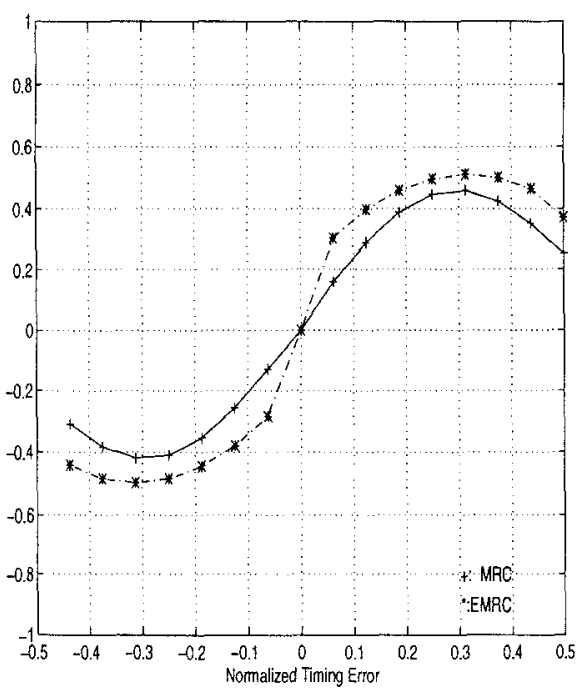

(1)

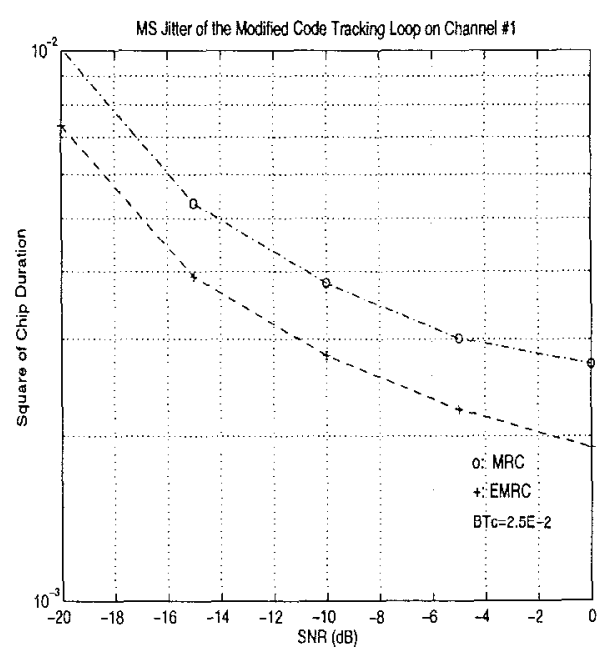

(2)

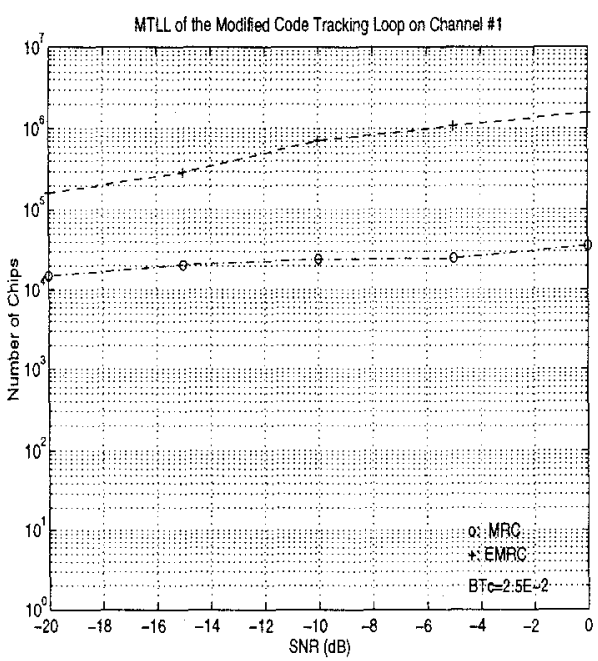

(3)

Fig. 2. (1)The S-curves, (2)Mean Square timing error, (3)The MTLL, of the modified code tracking loops with MRC and EMRC schemes on channel \#1 